\title{
PIPE DREAM COMPLEXES AND TRIANGULATIONS OF ROOT POLYTOPES BELONG TOGETHER
}

\author{
KAROLA MÉSZÁROS
}

\begin{abstract}
We show that the pipe dream complex associated to the permutation $1 n n-1 \cdots 2$ can be geometrically realized as a triangulation of the vertex figure of a root polytope. Leading up to this result we show that the Grothendieck polynomial specializes to the $h$-polynomial of the corresponding pipe dream complex, which in certain cases equals the $h$-polynomial of canonical triangulations of root (and flow) polytopes, which in turn equals a specialization of the reduced form of a monomial in the subdivision algebra of root (and flow) polytopes. Thus, we connect Grothendieck polynomials to reduced forms in subdivision algebras and root (and flow) polytopes. We also show that root polytopes can be seen as projections of flow polytopes, explaining that these families of polytopes possess the same subdivision algebra.
\end{abstract}

\section{Contents}

1. Introduction

2. Grothendieck polynomials

3. Reduced forms in the subdivision algebra

4. On the relation of root and flow polytopes 6

4.1. Root polytopes.

4.2. Flow polytopes. 8

4.3. Are root polytopes and flow polytopes the same? 8

5. Geometric realization of pipe dream complexes via root polytopes 9

Acknowledgements 11

References

\section{INTRODUCTION}

In this paper we journey from Grothendieck polynomials to geometric realizations of pipe dream complexes via root polytopes. On this journey we meet reduced forms of monomials in the subdivision algebra of root and flow polytopes, and root and flow polytopes themselves. While the connection between Grothendieck polynomials and pipe dream complexes is a well known one, the other objects in the above list are not universally thought of as tied to Grothendieck polynomials and pipe dream complexes. As this work will illustrate, they might indeed belong together.

Grothendieck polynomials represent K-theory classes on the flag manifold; they generalize Schubert polynomials, which in turn generalize Schur polynomials. We show that Grothedieck polynomials specialize to $h$-polynomials of pipe dream complexes. Since pipe dream complexes are known to be homeomorphic to balls (except in a trivial case), we get that their $h$-polynomials, and thus shifted specialized Grothedieck polynomials have nonnegative coefficients. Such property was first observed by Kirillov Kir12, who indicated that he had an algebraic proof in mind.

The author was partially supported by a National Science Foundation Grant (DMS 1501059). 


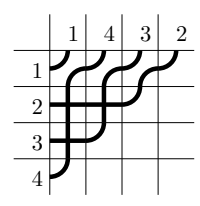

$x_{2}^{2} x_{3}$

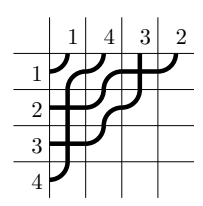

$x_{1} x_{2} x_{3}$

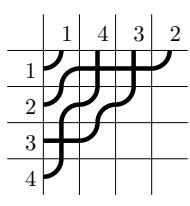

$x_{1}^{2} x_{3}$

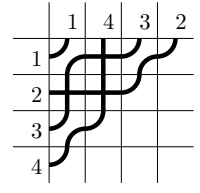

$x_{1} x_{2}^{2}$

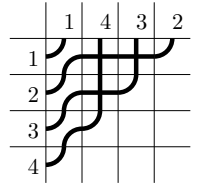

$x_{1}^{2} x_{2}$

Figure 1. All reduced pipe dreams for $w=1432$ (that is pipe dreams with exactly 3 crosses). The weights $w t_{x, y}(P)$ when $\mathbf{y}=\mathbf{0}$ are written below the reduced pipe dreams.

In Kir12] Kirillov also observed that a certain specialization of the shifted Grothendieck polynomial equals a specialization of a particular reduced form in the subdivision algebra of root and flow polytopes. His observation was based on numerical evidence. We explain this equality in terms of the geometry of the underlying pipe dream complex and root (and flow) polytopes. Indeed, we show that the mentioned pipe dream complex can be realized as the canonical triangulation of the vertex figure of the root polytope. No wonder then the specialized Grothendieck polynomial and reduced form are equal: they are the $h$-polynomial of the pipe dream complex and the $h$-polynomial of the canonical triangulation of the vertex figure of the root polytope, respectively. That the reduced form can be seen as the $h$-polynomial of the canonical triangulation of the flow polytope, and thus of the canonical triangulation of the vertex figure of the root polytope, was proved in [Mész14b]. The paper [Mész14a] also contains closely related results.

The outline of the paper is as follows. In Section 2 we show that the shifted $\beta$-Grothendieck polynomial corresponding to the permutation $w$ is the $h$-polynomial of pipe dream complex of $w$ denoted by $P D(w)$. In Section 3 we define the subdivision algebra and reduced forms and recall related results. We also allude to the connection of Grothendieck polynomials and reduced forms. In Section 4 we explain why the subdivision algebras of root and flow polytopes are the same, by showing that root polytopes are projections of flow polytopes. Finally, in Section 5 we tie all the above together, by showing that the pipe dream complex $P D(1 n n-1 \cdots 2)$ can be realized as a canonical triangulation of a vertex figure of a root polytope. $P D(1 n n-1 \cdots 2)$ has been realized previously via the classical associahedron [PP12, Ceb12, CLS14.

\section{Grothendieck Polynomials}

In this section we define Grothendieck polynomials and explain that they specialize to $h$-polynomials of certain simplicial complexes called pipe dream complexes. Since the pipe dream complex is homeomorphic to a ball, its $h$-polynomial has nonnegative coefficients. Therefore, we immediately obtain nonnegativity properties of Grothendieck polynomials, which were observed by Kirillov in Kir12].

There are several ways to express Grothendieck polynomials, and we will present the expression in terms of pipe dreams here. Given a permutation $w$ in the symmetric group $S_{n}$ it can be represented by a triangular table filled with +'s (crosses) and 'r's (elbows) such that (1) the pipes intertwine according to $w$ and (2) two pipes cross at most once. Such representations of $w$ are called reduced pipe dreams, see Figure 1. Pipe dreams are also known as RC-graphs, and the reduced pipe dreams of a permutation were shown to be connected by ladder and chute moves by Bergeron and Billey in BB93. To each reduced pipe dream we can associate the weight $w t_{x, y}(P):=\prod_{(i, j) \in \operatorname{cross}(P)}\left(x_{i}-y_{j}\right)$, with $\operatorname{cross}(P)$ being the set of positions where $P$ has a cross. Note that throughout the literature the definition of the weight varies; however, all results can be phrased using any one convention.

A nonreduced pipe dream for $w \in S_{n}$ is a triangular table filled with crosses and elbows so that (1) the pipes intertwine according to $w$ whereby if two pipes have already crossed previously 


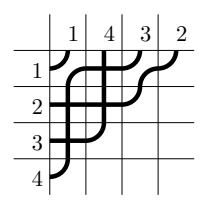

$x_{1} x_{2}^{2} x_{3}$

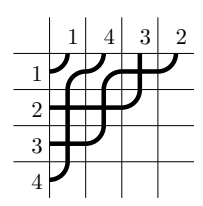

$x_{1} x_{2}^{2} x_{3}$

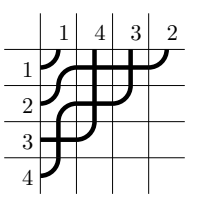

$x_{1}^{2} x_{2} x_{3}$

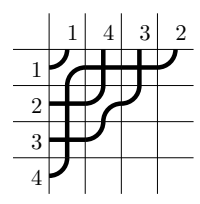

$x_{1}^{2} x_{2} x_{3}$

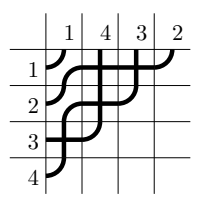

$x_{1}^{2} x_{2} x_{3}$

Figure 2. All pipe dreams with exactly 4 crosses for $w=1432$. The weights $w t_{x, y}(P)$ when $\mathbf{y}=\mathbf{0}$ are written below the pipe dreams.

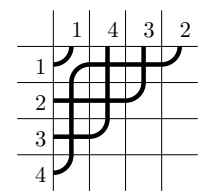

$$
x_{1}^{2} x_{2}^{2} x_{3}
$$

Figure 3. The unique pipe dreams with exactly 5 crosses for $w=1432$. The weights $w t_{x, y}(P)$ when $\mathbf{y}=\mathbf{0}$ are written below the pipe dream.

then we simply ignore the extra crossings and (2) there are two pipes that cross at least twice. All nonreduced pipe dreams for 1432 with a total of 4 crosses can be seen in Figure 2 and the unique pipe dreams for 1432 with a total of 5 crosses can be seen in Figure 3. We associate a weight $w t_{x, y}(P)$ to each pipe dream as above.

The set Pipes $(w)$, which is the set of all pipe dreams of $w$ (both reduced and nonreduced), naturally labels the interior simplices of the pipe dream complex $P D(w)$ associated to a permutation $w \in S_{n}$; see Figure 4 for $P D(1432)$. The pipe dream complex $P D(w)$ is a special case of a subword complex and can be defined as follows. A word of size $m$ is an ordered sequence $Q=\left(\sigma_{1}, \ldots, \sigma_{m}\right)$ of elements from the simple reflections $\left\{s_{1}, \ldots, s_{n-1}\right\}$ in $S_{n}$. An ordered subsequence $R$ of $Q$ is called a subword of $Q$. A subword $R$ of $Q$ represents $w \in S_{n}$ if the ordered product of simple reflections in $R$ is a reduced decomposition for $w$. The word $R$ contains $w \in S_{n}$ if some subsequence of $R$ represents $w$. The pipe dream complex $P D(w)$ is the set of subwords $\left(s_{n-1}, s_{n-2}, \ldots, s_{1}, s_{n-1}, s_{n-2}, \ldots, s_{2}, s_{n-1}, s_{n-2}, \ldots, s_{3}, \ldots, s_{n-1}, s_{n-2}, s_{n-1}\right) \backslash R$ whose complements $R$ contain $w$. The word $Q=\left(s_{n-1}, s_{n-2}, \ldots, s_{1}, s_{n-1}, s_{n-2}, \ldots, s_{2}, s_{n-1}, s_{n-2}, \ldots, s_{3}, \ldots\right.$, $\left.s_{n-1}, s_{n-2}, s_{n-1}\right)$ is called the triangular word.

The following theorem provides a combinatorial way of thinking about double Grothendieck polynomials.

Theorem 2.1. KMM4, FK94 The double Grothendieck polynomial $\mathfrak{G}_{w}(\mathbf{x}, \mathbf{y})$ for $w \in S_{n}$, where $\mathbf{x}=\left(x_{1}, \ldots, x_{n-1}\right)$ and $\mathbf{y}=\left(y_{1}, \ldots, y_{n-1}\right)$ can be written as

$$
\mathfrak{G}_{w}(\mathbf{x}, \mathbf{y})=\sum_{P \in \operatorname{Pipes}(w)}(-1)^{\operatorname{codim}_{P D(w)} F(P)} w t_{x, y}(P),
$$

where Pipes $(w)$ is the set of all pipe dreams of $w$ (both reduced and nonreduced), $F(P)$ is the interior face in $P D(w)$ labeled by the pipe dream $P$, $\operatorname{codim}_{P D(w)} F(P)$ denotes the codimension of $F(P)$ in $P D(w)$ and $w t_{x, y}(P)=\prod_{(i, j) \in \operatorname{cross}(P)}\left(x_{i}-y_{j}\right)$, with $\operatorname{cross}(P)$ being the set of positions where $P$ has a cross.

In the spirit of Theorem 2.1, we use the following definition for the double $\beta$-Grothendieck polynomial: 


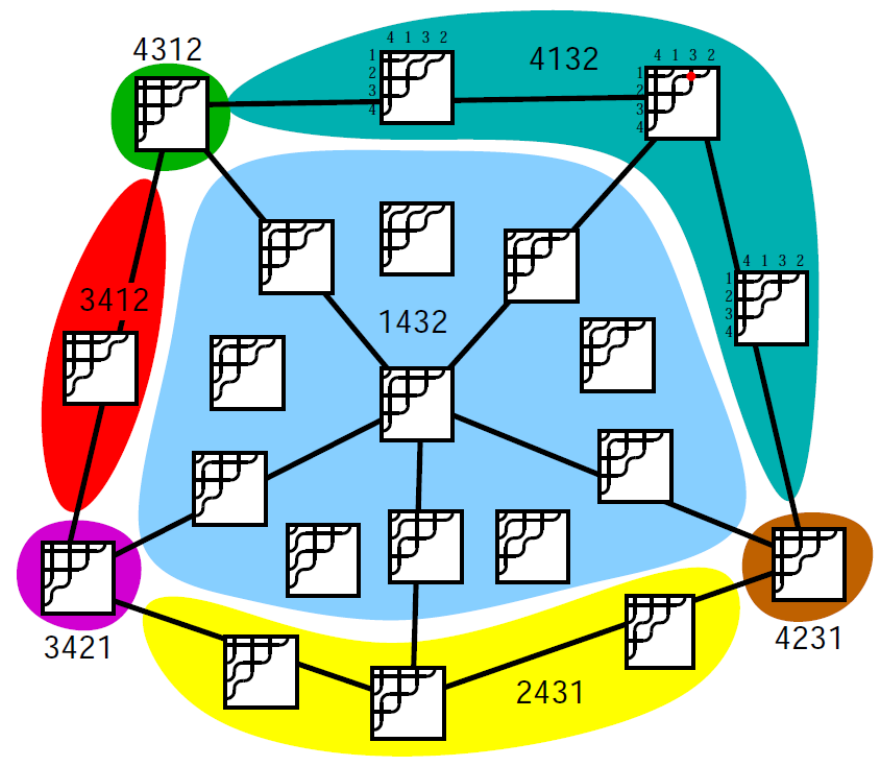

Figure 4. The pipe dream complex PD(1432). Figure used with permission from Knu04.

$$
\mathfrak{G}_{w}^{\beta}(\mathbf{x}, \mathbf{y})=\sum_{P \in \operatorname{Pipes}(w)} \beta^{\operatorname{codim}_{P D(w)} F(P)} w t_{x, y}(P)
$$

Note that if we assume that $\beta$ has degree -1 , while all other variables are of degree 1 , then the powers of $\beta$ 's simply make the polynomial $\mathfrak{G}_{w}^{\beta}(\mathbf{x}, \mathbf{y})$ homogeneous. We chose this definition of $\beta$-Grothendieck polynomials, as it will be the most convenient notationwise for our purposes.

Next we state a special case of $(2.2)$, since it will play a special role in this section.

Lemma 2.2. Denoting $\mathfrak{G}_{w}^{\beta}(\mathbf{x}, \mathbf{y})$ by $\mathfrak{G}_{w}^{\beta}(q, t)$ when we set all components of $\mathbf{x}$ to $q$ and all components of $\mathbf{y}$ to $t$, we have

$$
\mathfrak{G}_{w}^{\beta}(q, t)=(q-t)^{l(w)} \sum_{P \in \operatorname{Pipes}(w)}[\beta(q-t)]^{\operatorname{codim}_{P D(w)} F(P)},
$$

where $l(w)$ is the length of the permutation $w, F(P)$ is the interior face in $P D(w)$ labeled by the pipe dream $P$ and codim $_{P D(w)} F(P)$ denotes the codimension of $F(P)$ in $P D(w)$.

Proof. By (2.2) we have that

$$
\mathfrak{G}_{w}^{\beta}(q, t)=\sum_{P \in \operatorname{Pipes}(w)} \beta^{\operatorname{codim}_{P D(w)} F(P)}(q-t)^{|\operatorname{cross}(P)|} .
$$

Since the number of crosses in a pipe dream $P$ is $l(w)+\operatorname{codim}_{P D(w)} F(P)$, equation 2.3 follows.

The next lemma follows from the well-known relation between $f$ - and $h$-polynomials. We note that we take $h(\mathcal{C}, x)=\sum_{i=0}^{d} h_{i} x^{i}$ to be the $h$-polynomial of a $(d-1)$-dimensional simplicial complex $\mathcal{C}$. For a direct proof of the lemma see [Mész14b]. 
Lemma 2.3. [Sta96] Let $\mathcal{C}$ be a $(d-1)$-dimensional simplicial complex homeomorphic to a ball and $f_{i}^{\circ}$ be the number of interior faces of $\mathcal{C}$ of dimension $i$. Then

$$
h(\mathcal{C}, \beta+1)=\sum_{i=0}^{d-1} f_{i}^{\circ} \beta^{d-1-i}
$$

Using that the interior simplices of $P D(w)$ are in bijection with pipe dreams of $w$ we obtain the following corollary of Lemma 2.3

Corollary 2.4. Given $w \in S_{n}$ we have

$$
h(P D(w), \beta+1)=\sum_{P \in \operatorname{Pipes}(w)} \beta^{\operatorname{codim}_{P D(w)} F(P)},
$$

where $F(P)$ is the interior face in $P D(w)$ labeled by the pipe dream $P$ and $\operatorname{codim}_{P D(w)} F(P)$ denotes the codimension of $F(P)$ in $P D(w)$.

Finally, we obtain the following as a corollary of the above.

Theorem 2.5. We have

$$
\mathfrak{G}_{w}^{\beta-1}(q, q-1)=h(P D(w), \beta),
$$

where $h(P D(w), \beta)$ is the h-polynomial of $P D(w)$. In particular we have that $\mathfrak{G}_{w}^{\beta-1}(q, q-1) \in$ $\mathbb{Z}_{\geq 0}[\beta]$.

Proof. Corollary 2.4 yields $h(P D(w), \beta+1)=\sum_{P \in \operatorname{Pipes}(w)} \beta^{\operatorname{codim}_{P D(w)} F(P)}$. Together with Lemma 2.2 applied when $t=q-1$ we get 2.7 in Theorem 2.5. The nonnegativity of the coefficients of $\mathfrak{G}_{w}^{\beta-1}(q, q-1)$ then follows because of the nonnegativity of the $h$-polynomial of a simplicial complex which is homeomorphic to a ball. Recall that $P D(w)$ is known to be homeomorhpic to a ball, except in the trivial case when it is a $(-1)$-sphere, which case can be checked separately.

The nonnegativity of the coefficients of $\mathfrak{G}_{w}^{\beta-1}(1,0)$ was observed by Kirillov [Kir12]. Equation (2.7) makes clear why this is the case: because it is the $h$-polynomial of a simplicial complex which is homeomorphic to a ball, implying that its coefficients are nonnegative [Sta96].

\section{REDUCED FORMS IN THE SUBDIVISION ALGEBRA}

In this section we point to a connection between reduced forms in the so called subdivision algebra and Grothendieck polynomials. In Section 5 we provide a geometric realization of the pipe dream complex $P D(1 n(n-1) \ldots 2)$ via a triangulation of a root (or flow) polytope, which implies this connection. In Section 4 we explain the connection between root and flow polytopes via the subdivision algebra also explaining the algebra's name.

The subdivision algebra $\mathcal{S}(\beta)$ is a commutative algebra generated by the variables $x_{i j}, 1 \leq$ $i<j \leq n$, over $\mathbb{Q}[\beta]$, subject to the relations $x_{i j} x_{j k}=x_{i k}\left(x_{i j}+x_{j k}+\beta\right)$, for $1 \leq i<j<k \leq n$. This algebra is called the subdivision algebra, because its relations can be seen geometrically as subdividing flow and root polytopes. This is explained in detail in Section 4 . The subdivision algebra has been used extensively for subdividing root and flow polytopes in [Mész15, MM15, Mész14b, Mész14a, Mész11a, Mész11b].

A reduced form of the monomial in the algebra $\mathcal{S}(\beta)$ is a polynomial obtained by successively substituting $x_{i k}\left(x_{i j}+x_{j k}+\beta\right)$ in place of an occurrence of $x_{i j} x_{j k}$ for some $i<j<k$ until no further reduction is possible. Note that the reduced forms are not necessarily unique. 
by

A possible sequence of reductions in algebra $\mathcal{S}(\beta)$ yielding a reduced form of $x_{12} x_{23} x_{34}$ is given

$$
\begin{aligned}
x_{12} \boldsymbol{x}_{\mathbf{2 3}} \boldsymbol{x}_{\mathbf{3 4}} \rightarrow & \boldsymbol{x}_{\mathbf{1 2}} x_{24} \boldsymbol{x}_{\mathbf{2 3}}+\boldsymbol{x}_{\mathbf{1 2}} x_{34} \boldsymbol{x}_{\mathbf{2 4}}+\beta \boldsymbol{x}_{\mathbf{1 2}} \boldsymbol{x}_{\mathbf{2 4}} \\
\rightarrow & \boldsymbol{x}_{\mathbf{2 4}} x_{13} \boldsymbol{x}_{\mathbf{1 2}}+x_{24} x_{23} x_{13}+\beta x_{24} x_{13}+x_{34} x_{14} x_{12}+x_{34} x_{24} x_{14} \\
& +\beta x_{34} x_{14}+\beta x_{14} x_{12}+\beta x_{24} x_{14}+\beta^{2} x_{14} \\
\rightarrow & x_{13} x_{14} x_{12}+x_{13} x_{24} x_{14}+\beta x_{13} x_{14}+x_{24} x_{23} x_{13}+\beta x_{24} x_{13} \\
& +x_{34} x_{14} x_{12}+x_{34} x_{24} x_{14}+\beta x_{34} x_{14}+\beta x_{14} x_{12}+\beta x_{24} x_{14} \\
& +\beta^{2} x_{14}
\end{aligned}
$$

where the pair of variables on which the reductions are performed is in boldface. The reductions are performed on each monomial separately.

Given a graph $G$, denote by $Q_{G}(\beta)$ the reduced form of the monomial $\prod_{(i, j) \in E(G)} x_{i j}$ specialized at $x_{i j}=1$ for all $1 \leq i<j \leq n$. The polynomial $Q_{G}(\beta)$ is unique, though the reduced form with variables $x_{i j}$ is not Mész11a. In recent work Mész14b the author connected $Q_{G}(\beta)$ to the $h$-polynomials of triangulations of flow polytopes of $G=(V(G) \cup\{s, t\}, E(G) \cup\{(s, i),(i, t) \mid i \in$ $V(G)\})$. Flow polytopes are defined in Section 4.2 ; in the next theorem we treat their triangulations, which we denote by $\mathcal{C}$, as a simplicial complex. Since $\mathcal{C}$ is a simplicial complex homeomorphic to a ball, it follows that the $h$-polynomial of a triangulation of a polytope has nonnegative coefficients [Sta96].

Theorem 3.1. [Mész14b] For any graph $G$ we have

$$
Q_{G}(\beta)=h(\mathcal{C}, \beta+1),
$$

where $\mathcal{C}$ is any unimodular triangulation of the flow polytope $\mathcal{F}_{\tilde{G}}(1,0, \ldots, 0,-1)$ and $h(\mathcal{C}, x)$ is its h-polynomial. In particular, the reduced form $Q_{G}(\beta-1)$ is a polynomial in $\beta$ with nonnegative coefficients.

For brevity, use the notation $\mathfrak{G}_{w}(\beta)$ for $\mathfrak{G}_{w}^{\beta}(1,0)$, the double $\beta$-Grothendieck polynomial evaluated when all $x$ 's are set to 1 and $y$ 's are set to 0 . In this notation Theorem 2.5 specialized at $q=1$ states that $\mathfrak{G}_{w}(\beta)=h(P D(w), \beta+1)$.

Note the similarity of the statements of Theorems 3.1 and 2.5 as a certain polynomial equaling the $h$-polynomial of a simplicial complex. Paired with Kirillov's observation in Kir12, Proposition 3.1] that

$$
Q_{P_{n}}(\beta)=\mathfrak{G}_{\pi}(\beta),
$$

for the permutation $\pi=1 n n-1 \cdots 2$ and path graph $P_{n}=([n],\{(i, i+1) \mid i \in[n-1]\})$, we obtain that $h(P D(\pi), \beta)=h(\mathcal{C}, \beta)$, where $\mathcal{C}$ is any unimodular triangulation of the flow polytope $\mathcal{F}_{\tilde{P}_{n}}$. The previous raises the natural question: can $P D(\pi)$ be realized geometrically as a triangulation $\mathcal{C}$ of the flow polytope $\mathcal{F}_{\tilde{P}_{n}}$ ? The answer is almost yes as we explain in the next sections.

\section{ON THE RELATION OF ROOT AND FLOW POLYTOPES}

This section explains the geometric reasons for root and flow polytopes to have the same subdivision algebras and in turn to possess dissections with identical descriptions via reduced forms Mész15, MM15, Mész14b, Mész14a, Mész11a, Mész11b]. The simplest reason for the above would be if root and flow polytopes were equivalent. While this is not the case, the truth does not lie far from it, as we will see. 
4.1. Root polytopes. In the terminology of [Pos09], a root polytope of type $A_{n}$ is the convex hull of the origin and some of the points $e_{i j}^{-}:=e_{i}-e_{j}$ for $1 \leq i<j \leq n+1$, where $e_{i}$ denotes the $i^{t h}$ coordinate vector in $\mathbb{R}^{n+1}$. A very special root polytope is the full root polytope

$$
\mathcal{P}\left(A_{n}^{+}\right)=\operatorname{ConvHull}\left(0, e_{i j}^{-} \mid 1 \leq i<j \leq n+1\right),
$$

where $e_{i j}^{-}=e_{i}-e_{j}$. In this paper we restrict ourself to a class of root polytopes including $\mathcal{P}\left(A_{n}^{+}\right)$, which have subdivision algebras Mész11a.

Let $G$ be an acyclic graph on the vertex set $[n+1]$. Define

$$
\begin{gathered}
\mathcal{V}_{G}=\left\{e_{i j}^{-} \mid(i, j) \in E(G), i<j\right\}, \text { a set of vectors associated to } G ; \\
\mathcal{C}(G)=\left\langle\mathcal{V}_{G}\right\rangle:=\left\{\sum_{e_{i j}^{-} \in \mathcal{V}_{G}} c_{i j} e_{i j}^{-} \mid c_{i j} \geq 0\right\}, \text { the cone associated to } G ; \text { and } \\
\overline{\mathcal{V}}_{G}=\Phi^{+} \cap \mathcal{C}(G), \text { all the positive roots of type } A_{n} \text { contained in } \mathcal{C}(G),
\end{gathered}
$$

where $\Phi^{+}=\left\{e_{i j}^{-} \mid 1 \leq i<j \leq n+1\right\}$ is the set of positive roots of type $A_{n}$.

The root polytope $\mathcal{P}(G)$ associated to the acyclic graph $G$ is

$$
\mathcal{P}(G)=\operatorname{ConvHull}\left(0, e_{i j}^{-} \mid e_{i j}^{-} \in \overline{\mathcal{V}}_{G}\right)
$$

The root polytope $\mathcal{P}(G)$ associated to graph $G$ can also be defined as

$$
\mathcal{P}(G)=\mathcal{P}\left(A_{n}^{+}\right) \cap \mathcal{C}(G) .
$$

Note that $\mathcal{P}\left(A_{n}^{+}\right)=\mathcal{P}\left(P_{n+1}\right)$ for the path graph $P_{n+1}$ on the vertex set $[n+1]$.

We can view reduced forms in the subdivision algebra in terms of graphs, as hinted at in the previous section.

The reduction rule for graphs: Given a graph $G_{0}$ on the vertex set $[n+1]$ and $(i, j),(j, k) \in$ $E\left(G_{0}\right)$ for some $i<j<k$, let $G_{1}, G_{2}, G_{3}$ be graphs on the vertex set $[n+1]$ with edge sets

$$
\begin{aligned}
& E\left(G_{1}\right)=E\left(G_{0}\right) \backslash\{(j, k)\} \cup\{(i, k)\}, \\
& E\left(G_{2}\right)=E\left(G_{0}\right) \backslash\{(i, j)\} \cup\{(i, k)\}, \\
& E\left(G_{3}\right)=E\left(G_{0}\right) \backslash\{(i, j),(j, k)\} \cup\{(i, k)\} .
\end{aligned}
$$

We say that $G_{0}$ reduces to $G_{1}, G_{2}, G_{3}$ under the reduction rules defined by equations (4.3).

The reason for the name subdivision algebra is the following key lemma appearing in [Mész11a]:

Lemma 4.1. [Mész11a] (Reduction Lemma for Root Polytopes) Given an acyclic graph $G_{0}$ with $d$ edges, let $(i, j),(j, k) \in E\left(G_{0}\right)$ for some $i<j<k$ and $G_{1}, G_{2}, G_{3}$ as described by equations (4.3). Then

$$
\mathcal{P}\left(G_{0}\right)=\mathcal{P}\left(G_{1}\right) \cup \mathcal{P}\left(G_{2}\right)
$$

where all polytopes $\mathcal{P}\left(G_{0}\right), \mathcal{P}\left(G_{1}\right), \mathcal{P}\left(G_{2}\right)$ are d-dimensional and

$$
\mathcal{P}\left(G_{3}\right)=\mathcal{P}\left(G_{1}\right) \cap \mathcal{P}\left(G_{2}\right) \text { is }(d-1) \text {-dimensional. }
$$

What the Reduction Lemma really says is that performing a reduction on an acyclic graph $G_{0}$ is the same as dissecting the $d$-dimensional polytope $\mathcal{P}\left(G_{0}\right)$ into two $d$-dimensional polytopes $\mathcal{P}\left(G_{1}\right)$ and $\mathcal{P}\left(G_{2}\right)$, whose vertex sets are subsets of the vertex set of $\mathcal{P}\left(G_{0}\right)$, whose interiors are disjoint, whose union is $\mathcal{P}\left(G_{0}\right)$, and whose intersection is a facet of both. It is clear then that the reduced form can be seen as a dissection of the root polytope into simplices. 
4.2. Flow polytopes. Now we define flow polytopes and explain the analogue of the Reduction Lemma for them. Let $G$ be a loopless graph on the vertex set $[n+1]$, and let in $(e)$ denote the smallest (initial) vertex of edge $e$ and fin $(e)$ the biggest (final) vertex of edge $e$. Think of fluid flowing on the edges of $G$ from the smaller to the bigger vertices, so that the total fluid volume entering vertex 1 is one and leaving vertex $n+1$ is one, and there is conservation of fluid at the intermediate vertices. Formally, a flow $f$ of size one on $G$ is a function $f: E \rightarrow \mathbb{R}_{\geq 0}$ from the edge set $E$ of $G$ to the set of nonnegative real numbers such that

and for $2 \leq i \leq n$

$$
1=\sum_{e \in E, \operatorname{in}(e)=1} f(e)=\sum_{e \in E, \operatorname{fin}(e)=n+1} f(e)
$$

$$
\sum_{e \in E, \operatorname{fin}(e)=i} f(e)=\sum_{e \in E, \operatorname{in}(e)=i} f(e)
$$

The flow polytope $\mathcal{F}_{G}$ associated to the graph $G$ is the set of all flows $f: E \rightarrow \mathbb{R}_{\geq 0}$ of size one.

In this paper we restrict our attention to flow polytopes of certain augmented graphs $\tilde{G}=$ $(V(G) \cup\{s, t\}, E(G) \cup\{(s, i),(i, t) \mid i \in V(G)\}):$

Lemma 4.2. [Mész15, MM15] (Reduction Lemma for Flow Polytopes) Given a graph $G_{0}$ on the vertex set $[n+1]$ and $(i, j),(j, k) \in E\left(G_{0}\right)$, for some $i<j<k$, let $G_{1}, G_{2}, G_{3}$ be as in equations (4.3). Then

$$
\mathcal{F}_{\tilde{G}_{0}}=\mathcal{F}_{\tilde{G}_{1}} \bigcup \mathcal{F}_{\tilde{G}_{2}}
$$

where all polytopes $\mathcal{F}_{\tilde{G}_{0}}, \mathcal{F}_{\tilde{G}_{1}}, \mathcal{F}_{\tilde{G}_{2}}$, are of the same dimension and

$$
\mathcal{F}_{\tilde{G}_{3}}=\mathcal{F}_{\tilde{G}_{1}} \cap \mathcal{F}_{\tilde{G}_{2}} \text { is one dimension less. }
$$

4.3. Are root polytopes and flow polytopes the same? Given an acyclic graph $G$ Lemmas 4.1 and 4.2 imply that we can dissect $\mathcal{P}(G)$ and $\mathcal{F}_{\tilde{G}}$ with identical procedures. Are then $\mathcal{P}(G)$ and $\mathcal{F}_{\tilde{G}}$ equivalent for acyclic graphs $G$ ?

Note that the dimension of $\mathcal{P}(G)$ is $|E(G)|$, while the dimension of $\mathcal{F}_{\tilde{G}}$ is $|E(G)|+|V(G)|-1$, so the polytopes cannot be identical. However, we show that $\mathcal{F}_{\tilde{G}}$ can be projected onto an $|E(G)|-$ dimensional polytope $\mathcal{S}(G)$ that is equivalent to $\mathcal{P}(G)$. When with the subdivision algebra we are dissecting $\mathcal{P}(G)$ and $\mathcal{F}_{\tilde{G}}$ in identical ways, we get the corresponding (identifiable) induced dissections on $\mathcal{S}(G)$ and $\mathcal{P}(G)$.

Recall the well-known charaterization of the vertices of flow polytopes.

Lemma 4.3. [Sch03, Section 13.1a] The vertex set of $\mathcal{F}_{G}$ are the unit flows on increasing paths going from the smallest to the largest vertex of $G$.

The polytope $\mathcal{F}_{\tilde{G}}$ naturally lives in the space $\mathbb{R}^{|E(\tilde{G})|}$, with the coordinates corresponding to the edges of $\tilde{G}$. Denoting by $e_{(i, j)}$ the unit coordinate corresponding to the edge $(i, j) \in E(\tilde{G})$, we see that the vectors $e_{(i, j)},(i, j) \in E(G), e_{(s, i)}, e_{(i, t)}$, for $i \in[n]$, are an orthonormal basis of $\mathbb{R}^{|E(\tilde{G})|}$. Projecting onto the subspace $W$ of $\mathbb{R}^{|E(\tilde{G})|}$ spanned by $e_{(i, j)},(i, j) \in E(G)$, let the polytope $\mathcal{S}(G)$ be the image of $\mathcal{F}_{\tilde{G}}$. Denote the mentioned projection by $p$. The vertices of $\mathcal{S}(G)$ are 0 and vertices of the form $e_{\left(i_{1}, i_{2}\right)}+e_{\left(i_{2}, i_{3}\right)}+\cdots+e_{\left(i_{k}, i_{k+1}\right)}$, where $i_{1}<\cdots<i_{k+1},\left(i_{1}, i_{2}\right),\left(i_{2}, i_{3}\right), \ldots,\left(i_{k}, i_{k+1}\right) \in E(G)$.

Define the map $f: W \rightarrow \mathbb{R}^{n}$ as follows: $f\left(e_{(i, j)}\right)=e_{i}-e_{j}$, for $(i, j) \in E(G)$, and extend linearly. It follows by definition that the image of $\mathcal{S}(G)$ under $f$ is $\mathcal{P}(G)$. Since for an acyclic graph $G$ the vectors $e_{i}-e_{j},(i, j) \in E(G)$, are linearly independent, we get that $f$ is an affine map which is a bijection onto $\mathcal{P}(G)$ when restricted to $\mathcal{S}(G)$. Thus, $\mathcal{S}(G)$ and $\mathcal{P}(G)$ are affinely (and thus combinatorially) equivalent polytopes. 
Let $G_{0}$ be an acyclic graph, and let $G_{1}, G_{2}, G_{3}$ be as specified (4.3) . Then a check shows that $f\left(p\left(\mathcal{F}_{\widetilde{G_{i}}}\right)\right)=\mathcal{P}\left(G_{i}\right)$, for $i \in[3]$ and thus any dissection of $\mathcal{F}_{\widetilde{G}_{0}}$ that we obtain by repeated reductions as in Lemma 4.2 under the map $f \circ p$ yields a dissection of $\mathcal{P}\left(G_{0}\right)$ obtained by the same sequence of reductions as interpreted in Lemma 4.1 .

The above considerations prove the following theorem, which relates root and flow polytopes. The maps $p$ and $f$ are as defined above.

Theorem 4.4. The root polytope $\mathcal{P}(G)$ is equivalent to $\mathcal{S}(G)$, which is a projection of $\mathcal{F}_{\tilde{G}}$. Indeed, $\mathcal{P}(G)=f\left(p\left(\mathcal{F}_{\tilde{G}}\right)\right)$. Moreover, when the reductions (4.3) are performed on $G$ yielding dissections $\mathcal{D}_{1}$ and $\mathcal{D}_{2}$ of $\mathcal{P}(G)$ and $\mathcal{F}_{\tilde{G}}$, respectively, then $\mathcal{D}_{1}$ is the image of $\mathcal{D}_{2}$ under $f \circ p$.

It is in the sense of Theorem 4.4 that root polytopes and flow polytopes of acyclic graphs are the same. Since root polytopes are lower dimensional by definition and in this paper we are only concerned with acyclic graphs, namely, the path graph, we will use root polytopes in the rest of the paper.

\section{Geometric realization of pipe dream complexes via root polytopes}

The main theorem of this section is that the canonical triangulation of the vertex figure $\mathcal{V}\left(P_{n}\right)$ of $\mathcal{P}\left(P_{n}\right)$ at 0 is a geometric realization of the pipe dream complex $P D(1 n n-1 \cdots 2)$. The vertex figure of a polytope $P$ at vertex $v$ is the intersection of a hyperplane $\mathcal{H}$ with $P$, such that vertex $v$ is on one side of $\mathcal{H}$ and all the other vertices of $P$ are on the other side of $\mathcal{H}$. See [Zie95, p.54] for further details. We now explain the canonical triangulation of $\mathcal{P}\left(P_{n}\right)$; there is an analogous triangulation for all root (and flow) polytopes Mész11a, Mész14a, but since we are only concerned with $\mathcal{P}\left(P_{n}\right)$ in this section, we restrict our attention to this case. $P D(1 n n-1 \cdots 2)$ has previously been been realized via the classical associahedron PP12, Ceb12, CLS14.

Recall that a graph $G$ on the vertex set $[n]$ is said to be noncrossing if there are no vertices $i<j<k<l$ such that $(i, k)$ and $(j, l)$ are edges in $G$. A graph $G$ on the vertex set $[n]$ is said to be alternating if there are no vertices $i<j<k$ such that $(i, j)$ and $(j, k)$ are edges in $G$.

Theorem 5.1. [GGP97, Mész11a Let $T_{1}, \ldots, T_{k}$ be all the noncrossing alternating spanning trees of $K_{n}$. Then $\mathcal{P}\left(T_{1}\right), \ldots, \mathcal{P}\left(T_{k}\right)$ are top dimensional simplices in a triangulation of $\mathcal{P}\left(P_{n}\right)$. Moreover,

$$
\mathcal{P}\left(T_{i_{1}}\right) \cap \cdots \cap \mathcal{P}\left(T_{i_{l}}\right)=\mathcal{P}\left(T_{i_{1}} \cap \cdots \cap T_{i_{l}}\right),
$$

where $i_{1}, \ldots, i_{l} \in[k]$, and $T_{i_{1}} \cap \cdots \cap T_{i_{l}}=\left([n],\left\{(i, j) \mid(i, j) \in E\left(T_{i_{1}}\right) \cap \cdots \cap E\left(T_{i_{l}}\right)\right.\right.$.

The triangulation described in Theorem 5.1 is called the canonical triangulation of $\mathcal{P}\left(P_{n}\right)$. Since all top dimensional simplices in it contain 0 , we see that $\mathcal{V}\left(P_{n}\right)$ has a triangulation indexed by the same noncrossing alternating spanning trees:

Theorem 5.2. Let $T_{1}, \ldots, T_{k}$ be all the noncrossing alternating spanning trees of $K_{n}$. Then $\mathcal{P}\left(T_{1}\right) \cap$ $\mathcal{V}\left(P_{n}\right), \ldots, \mathcal{P}\left(T_{k}\right) \cap \mathcal{V}\left(P_{n}\right)$ are top dimensional simplices in a triangulation of $\mathcal{V}\left(P_{n}\right)$. Moreover,

$$
\mathcal{P}\left(T_{i_{1}}\right) \cap \cdots \cap \mathcal{P}\left(T_{i_{l}}\right) \cap \mathcal{V}\left(P_{n}\right)=\mathcal{P}\left(T_{i_{1}} \cap \cdots \cap T_{i_{l}}\right) \cap \mathcal{V}\left(P_{n}\right),
$$

where $i_{1}, \ldots, i_{l} \in[k]$, and $T_{i_{1}} \cap \cdots \cap T_{i_{l}}=\left([n],\left\{(i, j) \mid(i, j) \in E\left(T_{i_{1}}\right) \cap \cdots \cap E\left(T_{i_{l}}\right)\right.\right.$.

We call the triangulation described in Theorem 5.2 the canonical triangulation of $\mathcal{V}\left(P_{n}\right)$. The following is the main theorem of this section.

Theorem 5.3. The canonical triangulation of $\mathcal{V}\left(P_{n}\right)$ is a geometric realization of the pipe dream complex $P D(1 n n-1 \cdots 2)$. 


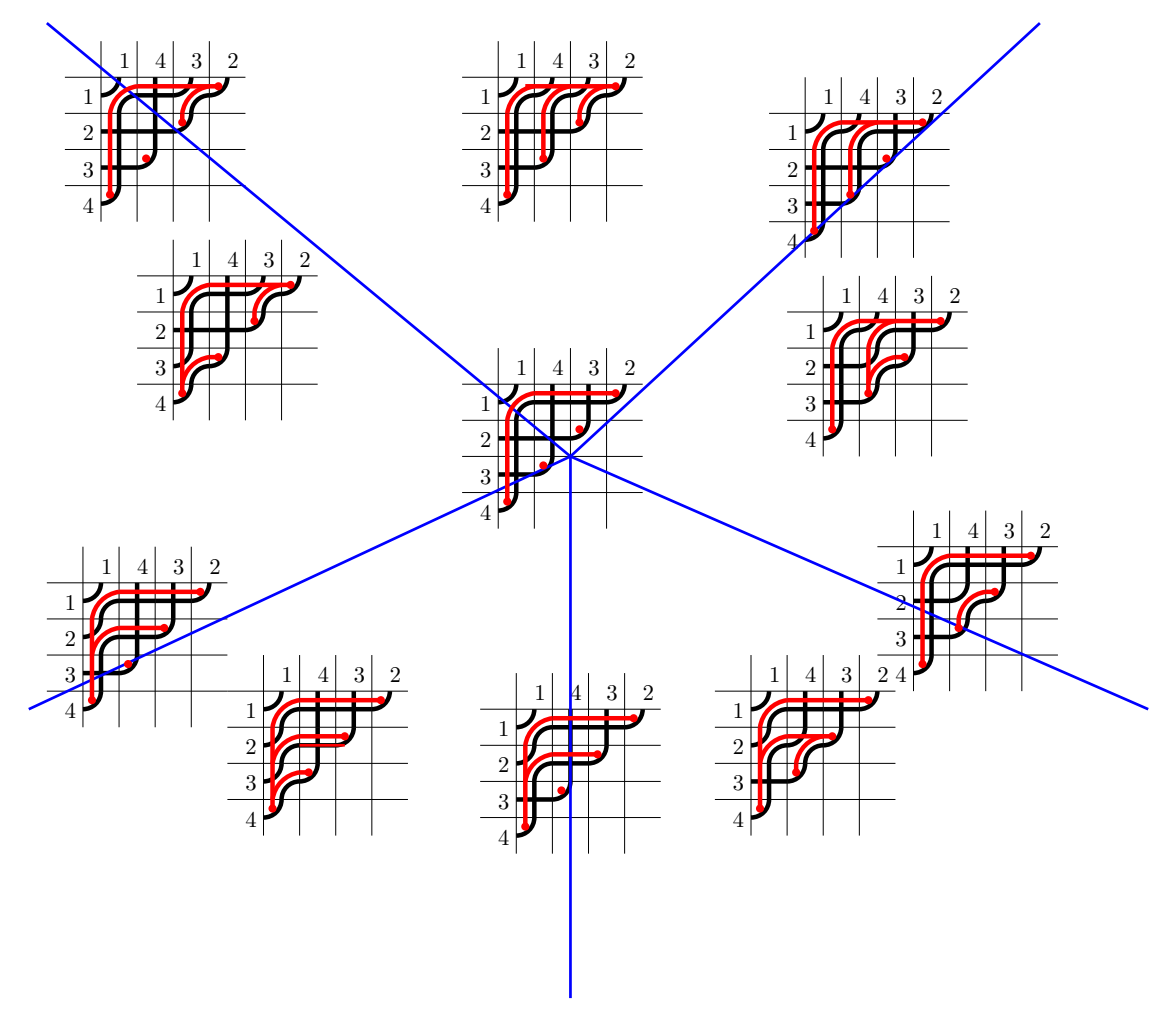

Figure 5. The interior simplices of $P D(1432)$ with the pipe dreams that label them. The graphs obtained via the bijection $G$ can be seen in red juxtaposed on top of the pipe dreams (rotated by $45^{\circ}$ ). Note that the top dimensional simplices are indeed labeled by the noncrossing alternating spanning trees of $K_{n}$.

Before proceeding to prove Theorem 5.3 we note that a proof of it could be obtained using the previous realization of $P D(1 n n-1 \cdots 2)$ via the associahedron. However, instead we will give a proof using root polytopes.

Proof of Theorem 5.3. First note that the dimensions of both $\mathcal{V}\left(P_{n}\right)$ and $P D(1 n n-1 \cdots 2)$ are $n-2$. Recall that the top dimensional simplices of $P D(1 n n-1 \cdots 2)$ are indexed by reduced pipe dreams of $1 n n-1 \cdots 2$, whereas their intersections by nonreduced pipe dreams in the following way. If we identify a pipe dream $P$ with its set of crosses, then the simplex at the intersection of the simplices labeled by pipe dreams $P_{i_{1}}, \ldots, P_{i_{l}}$ is labeled by a pipe dream $P_{i_{1}} \cup \cdots \cup P_{i_{l}}$, where in the latter we simply let the crosses be all the crosses in $P_{i_{1}}, \ldots, P_{i_{l}}$. See Figure 4 .

We first show that the top dimensional simplices in $P D(1 n n-1 \cdots 2)$ are in bijection with the top dimensional simplices in the canonical triangulation of $\mathcal{V}\left(P_{n}\right)$. Such a bijective map $G$ is easy to define. Given a reduced pipe dream $P$, let

$$
G(P)=([n],\{(i, j) \mid \text { there is an elbow in box }(n-j+1, i) \text { in } \mathrm{P}\}) .
$$

See Figure 5 for an example.

The map $G$ is clearly one-to-one. On the other hand we know that both reduced pipe dreams of $1 n n-1 \cdots 2$ and noncrossing alternating spanning trees of $K_{n}$ are counted by the Catalan numbers [Woo04 GGP97], thereby immediately yielding that $G$ is a bijection between the two sets. 
Given that the simplex at the intersection of the simplices labeled by pipe dreams $P_{i_{1}}, \ldots, P_{i_{l}}$ is labeled by a pipe dream $P_{i_{1}} \cup \cdots \cup P_{i_{l}}$ (as explained above), and

$$
\mathcal{P}\left(T_{i_{1}}\right) \cap \cdots \cap \mathcal{P}\left(T_{i_{l}}\right) \cap \mathcal{V}\left(P_{n}\right)=\mathcal{P}\left(T_{i_{1}} \cap \cdots \cap T_{i_{l}}\right) \cap \mathcal{V}\left(P_{n}\right),
$$

where $i_{1}, \ldots, i_{l} \in[k]$, and $T_{i_{1}} \cap \cdots \cap T_{i_{l}}=\left([n],\left\{(i, j) \mid(i, j) \in E\left(T_{i_{1}}\right) \cap \cdots \cap E\left(T_{i_{l}}\right)\right.\right.$ (as in Theorem 5.2) we have that the bijection $G$ extends to the lower dimensional interior simplices of $P D(1 n n-1 \cdots 2)$ and $\mathcal{V}\left(P_{n}\right)$. See Figure 5. Moreover, the same map also extends to the boundary simplices in the canonical triangulation of $\mathcal{V}\left(P_{n}\right)$ and those in $P D(1 n n-1 \cdots 2)$. Therefore, we can conclude that the canonical triangulation of $\mathcal{V}\left(P_{n}\right)$ is a geometric realization of $P D(1 n n-1 \cdots 2)$.

We remark that the $h$-vector of the canonical triangulation of $\mathcal{P}\left(P_{n}\right)$, and so also of $P D(1 n n-1 \cdots 2)$ consists of Narayana numbers; see [Sta99, Exercise 6.31b].

\section{ACKNOWLEDGEMENTS}

I am grateful to Allen Knutson for the many helpful discussions and references about topics related to this research. Special thanks to Sergey Fomin for an extensive conversation about double Grothendieck polynomials. I also thank Lou Billera and Ed Swartz for several valuable discussions and the anonymous referees for their comments.

\section{REFERENCES}

[BB93] N. Bergeron and S. Billey. RC-graphs and Schubert polynomials. Experiment. Math., 2(4):257-269, 1993.

[Ceb12] C. Ceballos. On associahedra and related topics. PhD thesis, Freie Universität Berlin, Berlin, 2012.

[CLS14] C. Ceballos, J-P. Labbé, and C. Stump. Subword complexes, cluster complexes, and generalized multiassociahedra. J. Algebraic Combin., 39(1):17-51, 2014.

[FK94] S. Fomin and A. N. Kirillov. Grothendieck polynomials and the Yang-Baxter equation. In Formal power series and algebraic combinatorics/Séries formelles et combinatoire algébrique, pages 183-189. DIMACS, Piscataway, NJ, 1994.

[GGP97] Israel M. Gelfand, Mark I. Graev, and Alexander Postnikov. Combinatorics of hypergeometric functions associated with positive roots. In The Arnold-Gelfand mathematical seminars, pages 205-221. Birkhäuser Boston, Boston, MA, 1997.

[Kir12] A.N. Kirillov. On some combinatorial and algebraic properties of Dunkl elements. RIMS preprint, 2012.

[KM04] A. Knutson and E. Miller. Subword complexes in Coxeter groups. Adv. Math., 184(1):161-176, 2004.

[Knu04] A. Knutson, 2004. Geometric vertex decompositions, FPSAC slides.

[Mész11a] K. Mészáros. Root polytopes, triangulations, and the subdivision algebra. I. Trans. Amer. Math. Soc., 363(8):4359-4382, 2011.

[Mész11b] K. Mészáros. Root polytopes, triangulations, and the subdivision algebra, II. Trans. Amer. Math. Soc., 363(11):6111-6141, 2011.

[Mész14a] K. Mészáros. $h$-polynomials of reduction trees. 2014. http://arxiv.org/abs/math/1019000arXiv:1407.2684.

[Mész14b] K. Mészáros. $h$-polynomials via reduced forms. 2014. http://arxiv.org/abs/math/1018992arXiv:1407.2685.

[Mész15] K. Mészáros. Product formulas for volumes of flow polytopes. Proc. Amer. Math. Soc., 143(3):937-954, 2015 .

[MM15] K. Mészáros and A. H. Morales. Flow polytopes of signed graphs and the Kostant partition function. Int. Math. Res. Not. IMRN, (3):830-871, 2015.

[Pos09] Alexander Postnikov. Permutohedra, associahedra, and beyond. Int. Math. Res. Not. IMRN, (6):10261106, 2009.

[PP12] Vincent Pilaud and Michel Pocchiola. Multitriangulations, pseudotriangulations and primitive sorting networks. Discrete Comput. Geom., 48(1):142-191, 2012.

[Sch03] Alexander Schrijver. Combinatorial optimization. Polyhedra and efficiency. Vol. A, volume 24 of Algorithms and Combinatorics. Springer-Verlag, Berlin, 2003.

[Sta96] R. Stanley. Combinatorics and Commutative Algebra. Birkhäuser, 1996.

[Sta99] Richard P. Stanley. Enumerative combinatorics. Vol. 2, volume 62 of Cambridge Studies in Advanced Mathematics. Cambridge University Press, Cambridge, 1999. With a foreword by Gian-Carlo Rota and appendix 1 by Sergey Fomin. 
[Woo04] A. Woo. Catalan numbers and schubert polynomials for $w=1(n+1) \cdots 2$. 2004 . http://arxiv.org/abs/math/0407160.

[Zie95] Günter M. Ziegler. Lectures on polytopes, volume 152 of Graduate Texts in Mathematics. Springer-Verlag, New York, 1995.

Karola Mészáros, Department of Mathematics, Cornell University, Ithaca Ny 14853 KAROLA@MATH.CORNELL.EDU 